\title{
Clinical heterogeneity of hypophysitis secondary to PD-1/PD-L1 blockade: insights from four cases
}

\author{
Isabella Lupi', Alessandro Brancatella1, Mirco Cosottini², Nicola Viola1, Giulia Lanzolla1, Daniele Sgrò, \\ Giulia Di Dalmazi³, Francesco Latrofa', Patrizio Caturegli4 and Claudio Marcocci1
}

${ }^{1}$ Endocrinology Unit, Department of Clinical and Experimental Medicine, University of Pisa, Pisa, Italy, ${ }_{2}^{2}$ Neuroradiology, Department of Translational Research and New Technologies in Medicine and Surgery, University of Pisa, Pisa, Italy, ${ }^{3 S e c t i o n}$ of Endocrinology, Department of Medicine and Aging Sciences, Ce.S.I-Me.T., "G.D'Annunzio" University of Chieti-Pescara, Chieti, Italy, and 4Division of Immunology, Department of Pathology، Johns Hopkins University, Baltimore Maryland, USA

Correspondence should be addressed to I Lupi

Email

isabellalupi@yahoo.it

\section{Summary}

Programmed cell death protein 1/programmed cell death protein ligand 1 (PD-1/PD-L1) and cytotoxic T-lymphocyte antigen 4/B7 (CTLA-4/B7) pathways are key regulators in T-cell activation and tolerance. Nivolumab, pembrolizumab (PD-1 inhibitors), atezolizumab (PD-L1 inhibitor) and ipilimumab (CTLA-4 inhibitor) are monoclonal antibodies approved for treatment of several advanced cancers. Immune checkpoint inhibitors (ICls)-related hypophysitis is described more frequently in patients treated with anti-CTLA-4; however, recent studies reported an increasing prevalence of anti-PD-1/PD-L1-induced hypophysitis which also exhibits slightly different clinical features. We report our experience on hypophysitis induced by anti-PD-1/anti-PD-L1 treatment. We present four cases, diagnosed in the past 12 months, of hypophysitis occurring in two patients receiving anti-PD-1, in one patient receiving anti-PD-1 and anti-CTLA-4 combined therapy and in one patient receiving anti-PD-L1. In this case series, timing, clinical presentation and association with other immune-related adverse events appeared to be extremely variable; central hypoadrenalism and hyponatremia were constantly detected although sellar magnetic resonance imaging did not reveal specific signs of pituitary inflammation. These differences highlight the complexity of ICl-related hypophysitis and the existence of different mechanisms of action leading to heterogeneity of clinical presentation in patients receiving immunotherapy.

\section{Learning points:}

- PD-1/PD-L1 blockade can induce hypophysitis with a different clinical presentation when compared to CTLA-4 blockade.

- Diagnosis of PD-1/PD-L1 induced hypophysitis is mainly made on clinical grounds and sellar MRI does not show radiological abnormalities.

- Hyponatremia due to acute secondary adrenal insufficiency is often the principal sign of PD-1/PD-L1-induced hypophysitis and can be masked by other symptoms due to oncologic disease.

- PD-1/PD-L1-induced hypophysitis can present as an isolated manifestation of irAEs or be in association with other autoimmune diseases 


\section{Background}

Immune checkpoint inhibitors (ICIs) are monoclonal antibodies directed against surface receptors involved in immune regulation such as cytotoxic T-lymphocyte antigen 4 (CLTA-4), programmed cell death protein 1 (PD-1), and programmed cell death protein ligand 1 (PD-L1). ICIs are now used in a variety of solid tumors where they cure a small percentage of patients, convert a deadly disease into a chronic one in about $20 \%$ of the patients, and remain ineffective in the remaining $80 \%$ (1). ICIs are also notable because they induce a broad spectrum of toxicities collectively referred to as immunerelated adverse events (irAEs). Like the primary forms of autoimmune diseases, irAEs can affect any organ or tissue in the body, although some sites such as skin, intestine, liver, lungs, and endocrine glands are preferentially targeted (2).

Hypophysitis caused by ICIs is seen most commonly in cancer patients treated with ipilimumab (anti-CTLA4) where it has a prevalence of about $12 \%$ (3). It is less common, instead, in cancer patients receiving anti-PD1 (nivolumab, pembrolizumab, cemiplimab) or anti-PDL1 (atezolizumab, avelumab, durvalumab), with a reported prevalence of about $0.5 \%$ (3). More and more cases of PD-1-related hypophysitis, however, are appearing in the literature, highlighting the uniqueness of this type of toxicity, the distinction with the CTLA4-related hypophysitis (3), and the overall heterogeneity of the clinical presentation and characteristics. We contribute four additional cases to expand the knowledge about this new condition.

\section{Case presentation}

\section{Case 1}

A 60-year-old man was treated with atezolizumab (anti-PD-L1: $1200 \mathrm{mg} / 3$ weeks) for his metastatic lung adenocarcinoma. After the fourth dose, he became rapidly ill and was diagnosed with ketoacidosis from type 1 diabetes mellitus. Intravenous administration of insulin corrected the hyperglycemia, but the hyponatremia and hyperkalemia remained.

\section{Case 2}

A 78-year-old man was treated with nivolumab (an anti-PD-1: $3 \mathrm{mg} / \mathrm{kg}$ every 2 weeks) for his nonsmall-cell lung cancer. After the sixth cycle, he developed nausea, vomiting, drowsiness, and fatigue requiring hospitalization. Laboratory exams showed hyponatremia (Table 1).

\section{Case 3}

A 80-year-old man received pembrolizumab (an anti-PD-1: $2 \mathrm{mg} / \mathrm{kg}$ every 3 weeks) for his metastatic melanoma. After the second cycle, he developed transient thyrotoxicosis followed by autoimmune hypothyroidism. Pembrolizumab was discontinued and replacement therapy with L-thyroxine was initiated. After about 4 months, pembrolizumab was restarted. Following seven additional cycles, the patient presented with headache and severe muscle weakness.

\section{Case 4}

A 43-year-old woman was initially treated with nivolumab (an anti-PD-1, $3 \mathrm{mg} / \mathrm{kg}$ every 2 weeks) for her metastatic melanoma. After the second cycle, she was diagnosed with transient thyrotoxicosis followed by autoimmune hypothyroidism requiring replacement therapy with L-thyroxine. After the fourth cycle, she developed vitiligo, and after the sixth cycle type 1 diabetes mellitus with severe diabetic ketoacidosis. Insulin therapy was started and ICIs treatment was interrupted. During the ensuing 4 months, due to progression of her melanoma, immunotherapy combining CTLA-4 and PD-1 blockade was started. Seven days after the first injection of ipilimumab and nivolumab, the patient developed fever and profound asthenia.

\section{Investigation}

\section{Case 1}

Laboratory assessment of pituitary function showed undetectable ACTH and cortisol levels also accompanied by hypogonadotropic hypogonadism (Table 1). Magnetic resonance imaging (MRI) of sellar region showed no evidence of metastasis or inflammation (Fig. 1A). Similarly, computed tomography (CT) scan of the abdomen excluded adrenal lesions. The presence of antibodies against 21-hydroxylase, as well as the markedly increased levels of renin (Table 1) suggested the existence of an autoimmune reaction directed also against the adrenal cortex. Antibodies against pancreatic beta cells were negative whereas those against the pituitary gland were found positive. Double immunofluorescence, performed as previously described using the patient serum and a commercial 
Table 1 Biochemical and hormonal features of patient series at the onset of hypophysitis.

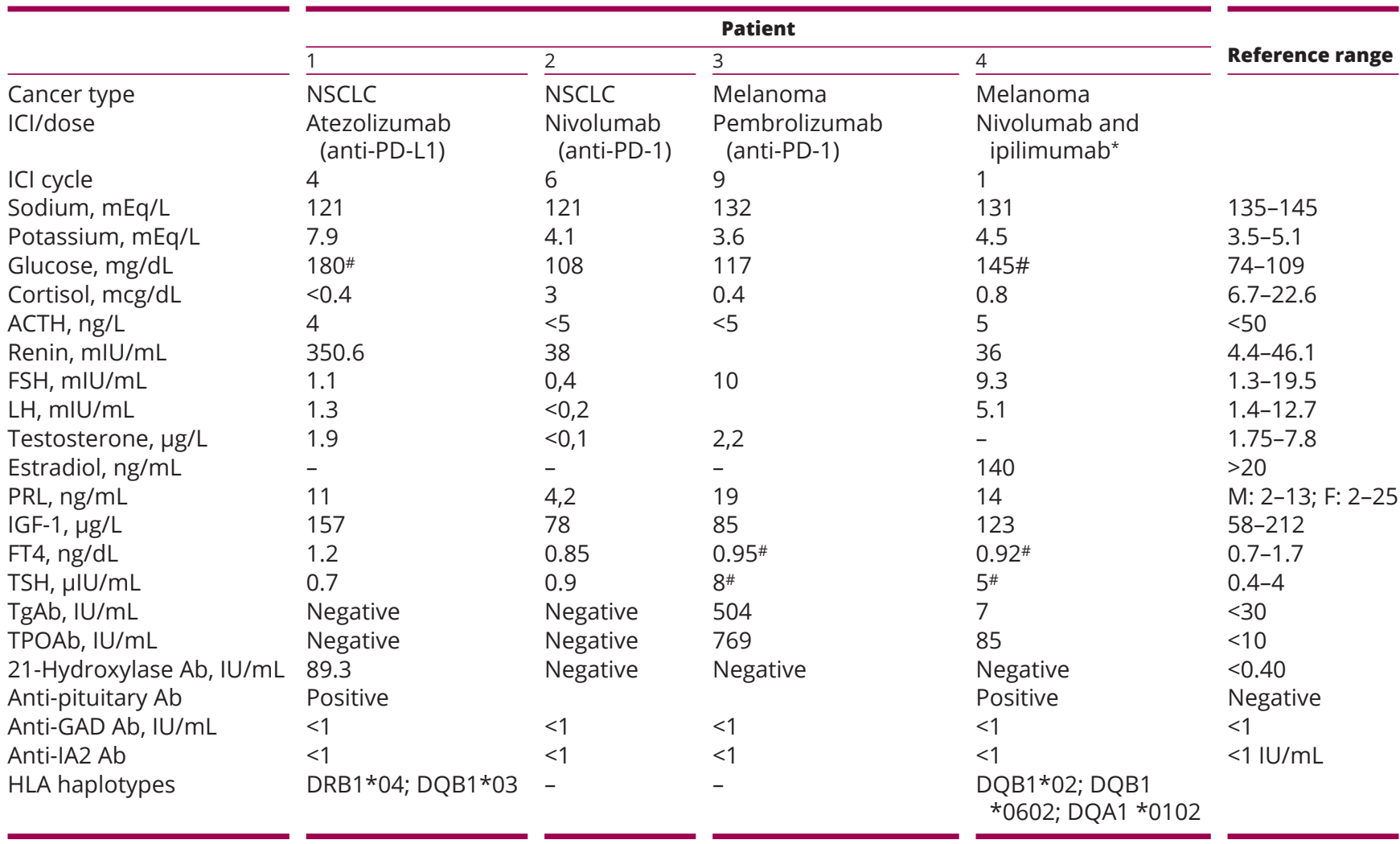

\#During L-thyroxine therapy, \#during insulin therapy. *Ipilimumab was given after six cycles of Nivolumab.

$\mathrm{ICl}$, immune checkpoint inhibitors.

antibody against GH, PRL, ACTH, LH, or TSH, showed a significant co-localization for TSH-secreting cells (data not shown) (4). Overall the administration of anti-PD-L1 triggered a polyglandular autoimmune syndrome (5).

\section{Case 2}

Adrenal insufficiency was suspected and confirmed by undetectable levels of cortisol and ACTH. Hormonal assessment showed, in addition to the ACTH deficiency, hypogonadotropic hypogonadism with undetectable levels of gonadotropins and testosterone (Table 1). Sellar MRI showed no lesions in the hypothalamic-pituitary region (Fig. 1B).

\section{Case 3}

Laboratory studies showed hyponatremia, and adrenal insufficiency secondary to isolated ACTH deficiency (Table 1 ). The thyroid function was normal under L-thyroxine (Table 1). Sellar MRI revealed no abnormalities in the anterior pituitary (Fig. 1C).

\section{Case 4}

Laboratory studies showed mild hyponatremia and secondary adrenal insufficiency (low levels of cortisol and ACTH, Table 1). Sellar MRI showed no signs of pituitary inflammation or mass (Fig. 1D). Organ-specific autoantibodies against 21-hydroxylase, transglutaminase, and glutamic decarboxylase were not found. On the contrary, antibodies against adeno-hypophyseal cells
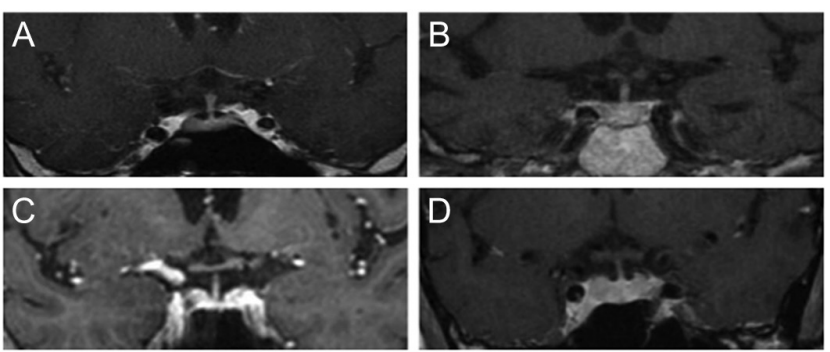

Figure 1

Sellar MRI of patient series at the onset of hypophysitis. T1-weighted images post-gadolinium show no signs of pituitary enlargement or other inflammatory signs. Panel A: patient 1. Panel B: patient 2. Panel C: patient 3. Panel D: patient 4. 
scattered throughout the gland were readily found, highlighting the cytosol in a homogenous pattern (Fig. $2 \mathrm{~A}$ and $\mathrm{B})$. Double immunofluorescence performed using the patient serum and a commercial antibody against GH, PRL, ACTH, LH, or TSH did not show significant co-localization (data not shown).

\section{Treatment}

\section{Case 1}

The patient was treated with insulin, hydrocortisone and fludrocortisone. Atezolizumab was withdrawn and conventional chemotherapy was began.

\section{Case 2}

The patient suspended nivolumab and began hydrocortisone replacement therapy.

\section{Case 3}

The patient began replacement therapy with hydrocortisone and continued L-thyroxine and pembrolizumab.

\section{Case 4}

Hormone replacement therapy with hydrocortisone was started, insulin, L-thyroxine and nivolumab and ipilimumab were continued.

\section{Outcome and follow-up}

\section{Case 1}

Tumor progression was documented on a CT scan 3 months later.

\section{Case 2}

Tumor progression was documented at CT scan after 3 months.

\section{Case 3}

No tumor progression was noted during the following 6 months.
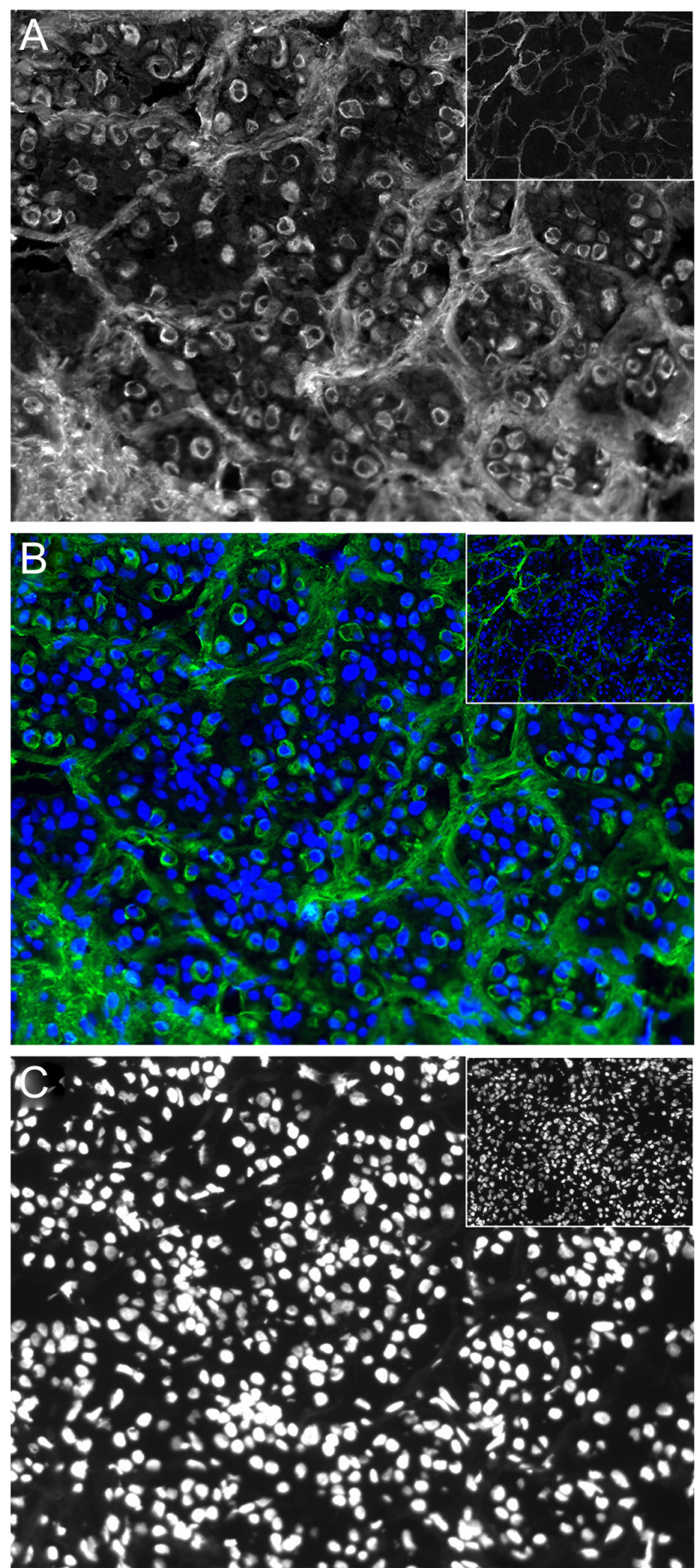

Figure 2

Assessment of serum pituitary antibodies by indirect

immunofluorescence in patient 4 . A normal human pituitary gland collected at autopsy was incubated with the patient serum (or healthy serum, insets). Panel A: staining obtained using the serum. Panel B: merged staining obtained using the serum and the DAPI (in blue). Panel C: DAPI staining. 


\section{Case 4}

Patient is continuing immunotherapy, no tumor progression was detected by whole body CT scan after 6 months.

\section{Discussion}

We report four cases of hypophysitis secondary to blockade of the PD-1 signaling pathway where hypophysitis manifested clinically at very different times after treatment initiation and was preceded or followed by other autoimmune manifestations in three out of four patients (Fig. 3). These differences highlight the complexity of the immune response and the existence of different mechanisms of action in patients receiving the same type of ICI.

Variations in irAEs prevalence across checkpoint inhibitors are likely due to their different mechanisms of action: a blockade of CTLA- 4 acts by enhancing T-cell priming, whereas a blockade of PD-1/PD-L1 is thought to act by enhancing a pre-existing CD8 T-cell response (6). Overall, ICIs can induce irAEs through several mechanisms by increasing the levels of pre-existing antibodies or inflammatory cytokines or, by increasing T-cell activity against antigens which are present in tumors as well as in healthy tissues (2). In the case of hypophysitis, an additional explanation for its high prevalence in patients treated by CTLA- 4 blockade is that, as reported in animal and human studies, CTLA-4 can be ectopically expressed in the pituitary gland, thus acting as an autoantigen when an anti-CTLA-4 agent is administered (7). As described in our four cases, symptoms of ICIs-related hypophysitis can be insidious and overlap those symptoms attributed to the oncologic disease, especially fatigue and headache.

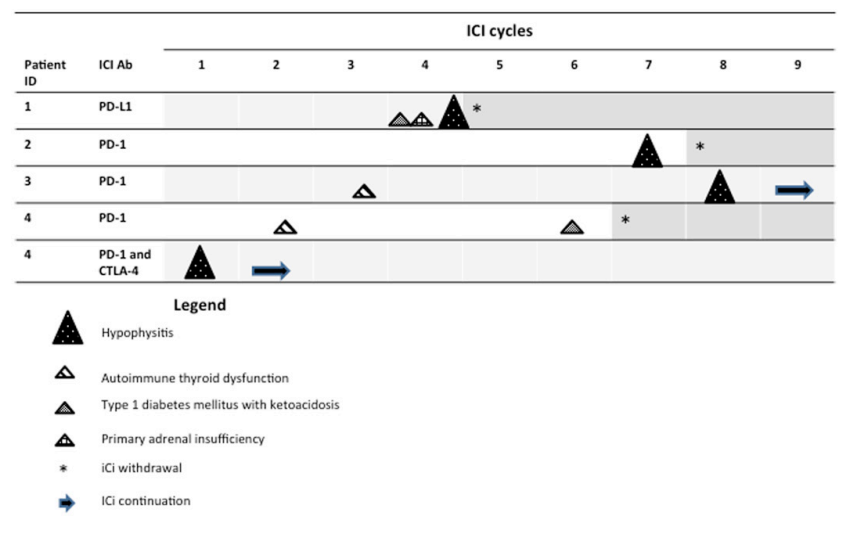

Figure 3

Timing of presentation of iRAEs in patient series.
Pituitary enlargement can be milder, or even transient and self-limiting, than that found in primary hypophysitis and appears to be almost exclusive of anti-CTLA-4 compared to anti-PD-1/PD-L1 therapy (3). Consequently, MRI may not be able to detect any specific sign of hypophysitis. Our population of four cases offers a spectrum of clinical presentation of hypophysitis; it presented over a timespan of 12-24 weeks after starting PD-1/PD-L1 blockade, and only after 1 week in one patient treated with double blockade PD-1 and CTLA-4. The main clinical feature was a symptomatic secondary adrenal insufficiency, also accompanied by other autoimmune manifestations, especially in cases 1 and 4 . In all four cases sellar MRI was negative for clear inflammatory signs or pituitary enlargement. Isolated ACTH defect has been found to be more prevalent in patients receiving anti-PD-1 therapy compared to those receiving anti-CTLA-4 (3), although the reason why ACTH-producing cells seem to be especially vulnerable to anti-PD-1 therapy is not yet known. Hyponatremia, a peculiar sign of hypoadrenalism secondary to ICIs related hypophysitis, was a common hallmark in all four cases. In central adrenal insufficiency, hyponatremia is mediated by increased hypothalamic secretion of CRH that, in turn, stimulates ADH and causes the inability to excrete free water. This condition mimics the syndrome of inappropriate antidiuresis (SIADH) that needs to be ruled out in oncologic patients - in which it may occur as a paraneoplastic syndrome - in order to initiate the correct treatment: fluid restriction in SIADH or corticosteroid replacement in adrenal insufficiency.

Hyponatremia is more frequently found in hypoadrenalism due to ICIs related hypophysitis than in lymphocytic (primary) hypophysitis (8). It is known that elderly patients have an enhanced release of $\mathrm{ADH}$ from the posterior pituitary which, when combined with adrenal insufficiency, contributes significantly to hyponatremia (9). We can also speculate that ICIs cause a sudden damage to ACTH-producing cells thus resulting in an acute adrenal insufficiency presenting with hyponatremia.

In our series the positivity of organ-specific antibodies, such as those found against pituitary or adrenal or thyroid gland, was also associated with organ damage. This finding is suggestive of an increased T cells activity and antibody production enhanced by ICIs.

Of notice, pituitary antibodies cannot be measured by a specific assay because the target autoantigen/s of autoimmune hypohysitis are not yet known. For this reason, at this time we can only rely on indirect immunofluorescence on human pituitary tissue to test pituitary autoimmunity. In keeping with other reports, 
pituitary antibodies that recognize TSH secreting cells are commonly found. The association of these antibodies with a functional defect of pituitary-thyroid axis, however, is not always demonstrated (10).

To test genetic susceptibility to autoimmune diseases, we performed analysis of human leukocyte antigen (HLA) haplotypes in the two patients (one and four) with polyglandular autoimmune syndrome. HLA typing confirmed genetic susceptibility for type 1 diabetes mellitus and Addison disease only in patient 1, whereas in patient 4 the analysis revealed haplotypes predisposing to celiac disease but protective for type 1 diabetes (11). These findings suggest that HLA typing is not a reliable tool to identify patients prone to develop irAEs in clinical practice.

In conclusion, ICIs-related hypophysitis appears to be not only a different clinical entity from lymphocytic hypophysitis but also presents different clinical features depending on the immune checkpoint used. It can present as an isolated manifestation of irAEs or be in association with other autoimmune diseases. To date, no clear genetic or clinical tool for predicting the onset of autoimmune manifestations during immunotherapy have been identified. Awareness of the heterogeneity in presentation of ICIs-related hypophysitis is crucial to guide oncologists and endocrinologists in daily clinical practice.

\section{Declaration of interest}

The authors declare that there is no conflict of interest that could be perceived as prejudicing the impartiality of this case report.

\section{Funding}

This work did not receive any specific grant from any funding agency in the public, commercial, or not-for-profit sector.

\section{Patient consent}

Written informed consent was obtained from the four patients for publication of this manuscript.

\section{Author contribution statement}

Isabella Lupi, Alessandro Brancatella and Patrizio Caturegli contributed to the final version of the manuscript. Giulia Di Dalmazi tested pituitary antibodies and prepared Fig. 2. All authors discussed the results and conclusions of this work.

\section{References}

1 Rogiers A, Boekhout A, Schwarze JK, Awada G, Blank CU \& Neyns B. Long-term survival, quality of life, and psychosocial outcomes in advanced melanoma patients treated with immune checkpoint inhibitors. Journal of Oncology 20192019 5269062. (https://doi. org/10.1155/2019/5269062)

2 Postow MA, Sidlow R \& Hellmann MD. Immune-related adverse events associated with immune checkpoint blockade. New England Journal of Medicine 2018378 158-168. (https://doi.org/10.1056/ NEJMra1703481)

3 Faje A, Reynolds K, Zubiri L, Lawrence D, Cohen J, Sullivan R, Nachtigall LB \& Tritos N. Hypophysitis secondary to nivolumab and pembrolizumab is a clinical entity distinct from ipilimumabassociated hypophysitis. European Journal of Endocrinology 2019181 211-219. (https://doi.org/10.1530/EJE-19-0238)

4 Ricciuti A, De Remigis A, Landek-Salgado MA, De Vincentiis L, Guaraldi F, Lupi I, Iwama S, Wand GS, Salvatori R \& Caturegli P. Detection of pituitary antibodies by immunofluorescence: approach and results in patients with pituitary diseases. Journal of Clinical Endocrinology and Metabolism 201499 1758-1766. (https://doi. org/10.1210/jc.2014-1049)

5 Lanzolla G, Coppelli A, Cosottini M, Del Prato S, Marcocci C \& Lupi I. Immune checkpoint blockade anti-PD-L1 as a trigger for autoimmune polyendocrine syndrome. Journal of the Endocrine Society 20193 496-503. (https://doi.org/10.1210/js.2018-00366)

6 Chen DS \& Mellman I. Elements of cancer immunity and the cancer-immune set point. Nature $2017541321-330$. (https://doi. org/10.1038/nature21349)

7 Caturegli P, Di Dalmazi G, Lombardi M, Grosso F, Larman HB, Larman T, Taverna G, Cosottini M \& Lupi I. Hypophysitis secondary to cytotoxic T-lymphocyte-associated protein 4 blockade: insights into pathogenesis from an autopsy series. American Journal of Pathology 2016186 3225-3235. (https://doi.org/10.1016/j. ajpath.2016.08.020)

8 Chang LS, Barroso-Sousa R, Tolaney SM, Hodi FS, Kaiser UB \& Min L. Endocrine toxicity of cancer immunotherapy targeting immune checkpoints. Endocrine Reviews 201940 17-65. (https://doi. org/10.1210/er.2018-00006)

9 Yatagai T, Kusaka I, Nakamura T, Nagasaka S, Honda K, Ishibashi S \& Ishikawa SE. Close association of severe hyponatremia with exaggerated release of arginine vasopressin in elderly subjects with secondary adrenal insufficiency. European Journal of Endocrinology 2003148 221-226. (https://doi.org/10.1530/eje.0.1480221)

10 Iwama S, De Remigis A, Callahan MK, Slovin SF, Wolchok JD \& Caturegli P. Pituitary expression of CTLA-4 mediates hypophysitis secondary to administration of CTLA-4 blocking antibody. Science Translational Medicine 20146 230ra45. (https://doi.org/10.1126/ scitranslmed.3008002)

11 Baisch JM, Weeks T, Giles R, Hoover M, Stastny P \& Capra JD. Analysis of HLA-DQ genotypes and susceptibility in insulindependent diabetes mellitus. New England Journal of Medicine 1990 322 1836-1841. (https://doi.org/10.1056/NEJM199006283222602)

Received in final form 11 September 2019 Accepted 20 September 2019 